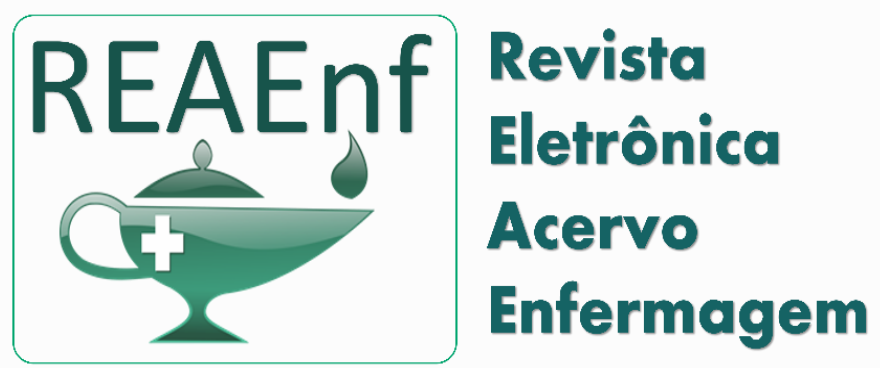

REVISÃO BIBLIOGRÁFICA

Recebido em: 8/2020

Aceito em: 9/2020

Publicado em: 11/2020

\title{
Exame retal digital: fatores relacionados à recusa do homem
}

\author{
Retal touch: related to refusal of man
}

\section{Examen rectal digital: factores relacionados con la negativa del hombre}

\author{
Suzana Leite de Oliveira ${ }^{1 *}$, Antero Machado Macêdo ${ }^{1}$, Naiadia Monteiro de Lima ${ }^{1}$, Paulo Rangel \\ Vieira Braga ${ }^{1}$, Gisele Reis Dias ${ }^{1}$.
}

\begin{abstract}
Resumo: Analisar os fatores relacionados à recusa do homem para a realização do exame a partir de revisão de literatura. Este estudo fundamenta-se em uma pesquisa integrativa, utilizando as bases de dados eletrônicas: Literatura Latino Americano em Ciências da Saúde (LILACS), Base de Dados em Enfermagem (BDENF), e Scientific Electronic Library Online (SCIELO). Da totalidade de 64 artigos reconhecidos nas bases, somente 5 correspondem às regras predeterminadas nesta revisão, estando 1 de forma repetida nas bases SCIELO e LILACS. Os fatores de recusa dos homens estão relacionados a aspectos culturais e emocionais nos quais estão atrelados à falta de informações que compromete a realização do toque. Percebeu-se nos estudos, que a falta de informação referente as vantagens de fazer o exame do toque retal, está ligada também a outros fatores levantados e averiguados na pesquisa, como o medo, o preconceito e a perda da masculinidade, que são consequências do desconhecimento deste importante exame.
\end{abstract}

Palavras-chave: Exame retal digital, Homem, Câncer de próstata.

\begin{abstract}
Analyze the factors related to man's refusal to perform the exam based on a literature review. This study is based on an integrative research, using the electronic databases: Latin American Literature in Health Sciences (LILACS), Nursing Database (BDENF), and Scientific Electronic Library Online (SCIELO). Of the total of 64 articles recognized in the databases, only 5 correspond to the predetermined rules in this review, 1 being repeated in the SCIELO and LILACS databases. The men 's refusal factors are related to cultural and emotional aspects in which they are linked to the lack of information that compromises the performance of the touch. It was noticed in the studies, that the lack of information regarding the advantages of examining the digital rectal exam is also linked to other factors raised and investigated in the research, such as fear, prejudice and the loss of masculinity, which are consequences of ignorance of this important examination.
\end{abstract}

Keywords: Digital retinal examination, Man, Prostate cancer.

Resumen: Analizar los factores relacionados con la negativa del hombre a realizar el examen a partir de una revisión de la literatura. Este estudio se basa en una investigación integradora, utilizando las bases de datos electrónicas: Literatura Latinoamericana en Ciencias de la Salud (LILACS), Base de Datos de Enfermería (BDENF) y Biblioteca Electrónica Científica en Línea (SCIELO). Del total de 64 artículos reconocidos en las bases de datos, solo 5 corresponden a las reglas predeterminadas en esta revisión, siendo 1 repetido en las bases de datos SCIELO y LILACS. Los factores de rechazo de los hombres están relacionados con aspectos culturales y emocionales en los que se vinculan a la falta de información que compromete la realización del toque. Se advirtió en los estudios, que la falta de información sobre las ventajas de examinar el tacto rectal también está vinculada a otros factores planteados e investigados en la investigación, como el miedo, los prejuicios y la pérdida de masculinidad, que son consecuencia de la ignorancia de este importante examen.

Palabras clave: Examen rectal digital, Hombre, Cáncer de próstata.

\footnotetext{
${ }^{1}$ Centro Universitário do Norte (UNINORTE), Manaus - AM. `E-mail: suzanaleite73@gmail.com
} 


\section{INTRODUÇÃO}

É primazia para a saúde do homem, que este obtenha uma qualidade de vida longa e saudável, por isso, o Ministério da Saúde (MS) fornece metas e diretrizes através da Política Nacional de Atenção Integral à Saúde do Homem (PNAISH), para aproximar este do cuidado pela sua vida, por intermédio da saúde pública. Esta política é baseada em cinco eixos básicos, e que são divididos em: acesso e acolhimento; saúde sexual e reprodutiva; paternidade e cuidado; doenças prevalentes, violências e acidentes; sendo possível identificar as alterações que afetam seu bem-estar, ajudando a promover uma assistência adequada por parte da equipe de saúde (BRASIL, 2009).

O câncer da próstata pertence às doenças e agravos não transmissíveis (DANT). Este câncer ocorre quando as células se tornam fora de ordem e ocasionam tumores malignos, e que são difíceis de controlar, por conseguinte, o câncer de próstata se manifesta. E o que dificulta mais o prognóstico, é que em algumas situações não há indícios, fazendo com que este indivíduo não perceba o descontrole da doença. No mundo, este representa o quarto tipo de câncer mais comum relacionado a mortes nos homens; no Brasil, é a segunda espécie que mais agride a população masculina. Geralmente ele ocorre em pacientes acima dos 50 anos, com prevalência acima dos 65 anos (INCA, 2018).

A identificação de mudanças na próstata é utilizada à dosagem do Antígeno Prostático Específico (PSA) onde é constatado através do sangue se o nível de PSA está acima de 4 nanogramas por mililitro $(\mathrm{ng} / \mathrm{mL})$. A ultrassonografia é também para detectar alteração do tamanho da próstata; e finalmente o exame retal digital, onde 0 avaliador consegue encontrar a próstata; e determinar se há alterações palpáveis, sendo considerado fidedigno por vários especialistas (SMELTZER SC e BARE BG, 2012).

O toque retal digital é apontado para perceber o câncer de próstata, no qual é feito para investigar formações tumorais e hipersensibilidade através da palpação da região anal. Mediante palpação da parede anterior dessa região é possível encontrar a próstata no homem, nota-se qualquer alteração como a sensibilidade a dor, presença de nódulos, consistência e avaliação do tamanho (BARROS AL, 2016).

Entretanto, os problemas na prevenção estão associados principalmente ao desconhecimento da população masculina, pois, esta é preconceituosa em relação à realização dos exames. $O$ homem não vai as consultas regularmente, e consequentemente, não realiza exames de rotina anualmente, delongando a descoberta da doença na formação do câncer. $\mathrm{Na}$ atenção primária não há ações rotineiras para a prevenção do câncer da próstata (SOUZA LM, et al., 2011).

O preconceito é um dos problemas que levam os homens a se negarem a fazer este exame. Esse procedimento interfere na imaginação masculina, no qual eles acreditam que o homem viril é obrigado a não demonstrar sentimentos, ou seja, não apresentar medo, fraqueza ou dor (SOUSA MCP, et al., 2014). Os aspectos figurativos devem ser considerados, pois estes estabelecem barreiras que implicam na sua masculinidade. Acredita-se que o homem é violado em sua perspectiva sobre qual é o papel do "macho", o que afeta em sua aceitação em fazer o exame retal digital, essa atitude representa para a maioria deles um obstante obstáculo para efetivar este procedimento (OLIVEIRA PSD, et al., 2015).

Embora o toque retal seja considerado um indicador de rastreamento para prevenir o câncer de próstata, quais fatores estão relacionados à recusa dos homens?

O interesse pelo estudo surgiu a partir das vivências acadêmicas na Atenção Básica, considerando que o câncer de próstata é o segundo tipo de câncer que mais acomete os homens, observa-se que no Brasil, a taxa de casos tem aumentado devido à melhoria dos métodos de diagnósticos, da divulgação das estatísticas e do aumento da população em idade avançada. Associada a esta razão, se quer também compreender por que um assunto tão importante como este, pode ser prejudicado devido à má compreensão do toque retal. Servirá também para esclarecer a sociedade sobre a necessidade de se vencer este mito, de que o homem que é submetido ao toque retal perde sua masculinidade.

Fora tentado levantar discussões e pontos que possam vir a contribuir com a comunidade acadêmica, somando tudo àquilo que já foi visto e abordado, com novas ideias, perspectivas e hipóteses que serão

REAEnf/EJNC | Vol. 4 | e5063 | DOI: https://doi.org/10.25248/REAenf.e5063.2020 Página 2 de 8 
apresentadas. Deste modo, temos como objetivo geral analisar os fatores relacionados à recusa do homem para a realização do exame, a partir de revisão de literatura.

\section{MÉTODOS}

Refere-se sobre uma Revisão Integrativa de Literatura. É o levantamento atual sobre uma ideia distintiva, que é conduzido de modo a identificar, analisar e sintetizar resultados. A revisão integrativa divide se em 6 fases: $1^{\underline{a}}$ Fase: elaboração da pergunta norteadora; $2^{\underline{a}}$ Fase: busca ou amostragem na literatura; $3^{\underline{a}}$ Fase:

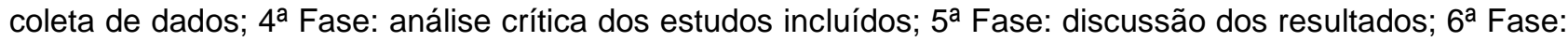
apresentação da revisão integrativa (SOUZA MT, et al., 2015).

Realizou-se discussão com a finalidade de identificar o problema, e chegamos em comum acordo: Quais são os fatores que estão relacionados à recusa dos homens?

Foram analisados e selecionados artigos nas bases de dados que se encontram na biblioteca virtual Scientific Electronic Library Online (SCIELO), Literatura Latino-Americana e do Caribe em Ciências da Saúde (LILACS) e Bases de Dados em Enfermagem (BDENF). Palavras-chave utilizados na SCIELO: Câncer de próstata e Recusa do Homem; na LILACS: Exame Retal Digital; e na BDENF: Exame Retal. Selecionamos com atenção e cautela os artigos nos meses de Março a maio de 2018.

Foram incluídos artigos que abordavam sobre o Exame Retal Digital, que esclarecesse as dificuldades do homem em realizar os exames de rastreamento do câncer de próstata, levando em consideração os últimos 8 anos de publicações relevantes para o tema, artigos nacionais e na íntegra. Os artigos excluídos tiveram como critérios ano de publicação anterior há 2010 , que não tivesse ajustado com os descritores e línguas estrangeiras.

A análise dos estudos foi realizada a partir de leitura flutuante. Definida como o contato fadigoso para dominar o assunto, compreendendo um primeiro contato que serão sujeitos à análise através das hipóteses e objetivos, para uma construção e organização do material por meio de explicação (CÂMARA RH, et al., 2013).

Foi utilizado para a apresentação e análise das literaturas um itinerário no modelo de fluxograma metodológico, e quadro representando os seguintes dados: $\mathrm{n}^{\circ}$, base, revista, título, autores, objetivo e ano, e mediante a discussão com clareza dos estudos e finalmente considerações finais.

\section{RESULTADOS E DISCUSSÃO}

$\mathrm{Na}$ presente pesquisa foram encontrados 64 artigos que se encontram na biblioteca virtual SCIELO, LILACS e BDENF no período de 2010 a 2018, e consequentemente, após leitura completa foram examinados e selecionados os artigos como se apresenta na Figura 1, através de fluxograma metodológico. Da totalidade de 64 artigos reconhecidos nas bases, somente 5 corresponderam as regras predeterminadas nesta revisão, estando 1 de forma repetida que podem ser encontradas nas bases SCIELO e LILACS. Foram elaborados para isto as seguintes categorias de acordo com o Quadro 1.

Nos textos selecionados mediante análise do objetivo, os resultados salientaram de acordo com a presente revisão integrativa. $O$ tipo de estudo que predominou foi do tipo exploratório e o descritivo, o tipo de abordagem que predominou foi o qualitativo, o período das publicações que prevaleceram foi o ano de 2015 no total de 3 artigos. Em relação às autorias dos artigos excluídos, eram de profissionais médicos, biomédicos, bioquímicos, sociólogos e enfermeiros.

Nas autorias dos artigos incluídos eram de: 1 médico graduado, 1 assistente social e sociólogo mestre, 9 enfermeiros graduados, 4 enfermeiros especialistas, 6 enfermeiros mestres e 3 enfermeiros doutores, com prevalência dos enfermeiros. Observamos que durante a pesquisa, são poucas as publicações que abordam sobre o tema. Isso diminuiu as informações sobre os fatores da recusa do homem em realizar 0 exame do toque retal. 
Figura 1 - Processo para seleção dos artigos.

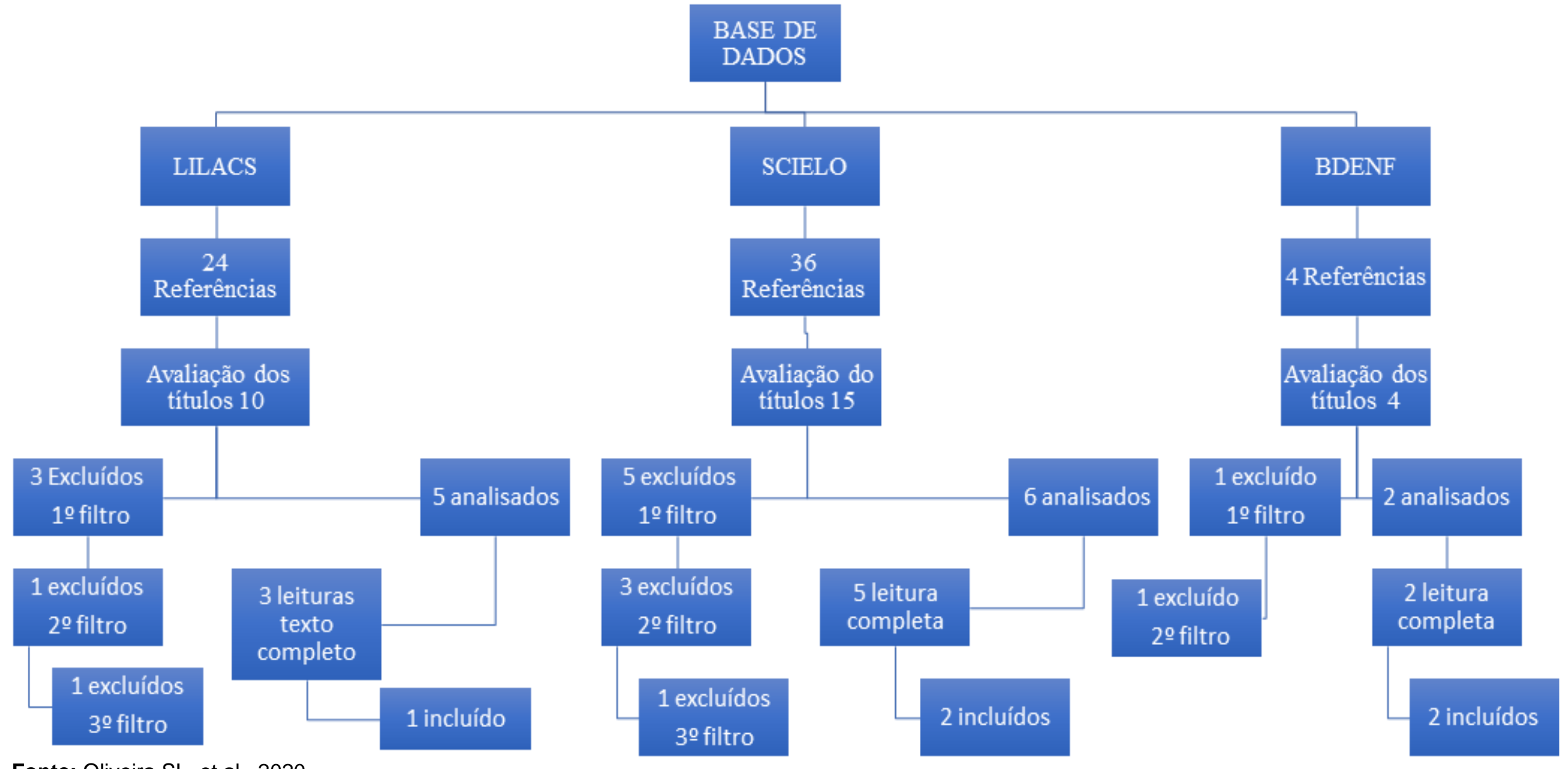

Fonte: Oliveira SL, et al., 2020. 
Quadro 1 - Caracterização dos artigos definidos para a revisão.

\begin{tabular}{|c|c|c|c|c|c|c|c|c|}
\hline № & Base & Revista & Título & Autor (es) & Objetivo & \multicolumn{2}{|c|}{ Métodos } & Ano \\
\hline 1 & BDENF & $\begin{array}{l}\text { Revista de } \\
\text { Enfermagem } \\
\text { UFPI }\end{array}$ & $\begin{array}{l}\text { Exame de toque retal: a } \\
\text { percepção de homens } \\
\text { quanto à sua realização }\end{array}$ & $\begin{array}{l}\text { Freitas MEM, et } \\
\qquad \text { al. }\end{array}$ & $\begin{array}{l}\text { Compreender a percepção dos homens } \\
\text { quanto à realização do exame de toque } \\
\text { retal, identificando os motivos pelos quais } \\
\text { não realizaram o exame e os sentimentos } \\
\text { que emergem nos homens que nunca se } \\
\text { submeteram ao exame de toque. }\end{array}$ & Descritivo & Qualitativa & 2015 \\
\hline 2 & BDENF & $\begin{array}{l}\text { Revista de } \\
\text { Enfermagem } \\
\text { UFPE }\end{array}$ & $\begin{array}{c}\text { Percepção dos Homens } \\
\text { Sobre o Exame Toque } \\
\text { Retal }\end{array}$ & $\begin{array}{l}\text { Oliveira PSD, et } \\
\quad \text { al. }\end{array}$ & $\begin{array}{l}\text { Compreender a percepção dos homens em } \\
\text { uma Estratégia de Saúde da Família quanto } \\
\text { à realização do exame de toque retal }\end{array}$ & $\begin{array}{l}\text { Interacionista } \\
\text { Simbólica e } \\
\text { Descritivo }\end{array}$ & Qualitativo & 2015 \\
\hline 3 & SCIELO & $\begin{array}{l}\text { Rev. Ciênc. } \\
\text { Saúde Nova } \\
\text { Esperança }\end{array}$ & $\begin{array}{l}\text { Toque Digital Prostático: } \\
\text { Conhecimento e } \\
\text { Resistência Masculina na } \\
\text { Terceira Idade }\end{array}$ & Ferreira DC, et al. & $\begin{array}{l}\text { Investigar o nível de conhecimento e a } \\
\text { resistência masculina, na terceira idade, ao } \\
\text { exame do toque digital prostático. }\end{array}$ & $\begin{array}{l}\text { Exploratória } \\
\text { descritiva }\end{array}$ & Quantitativa & 2015 \\
\hline 4 & SCIELO & $\begin{array}{c}\text { Revista } \\
\text { Interdisciplinar }\end{array}$ & $\begin{array}{c}\text { Aspectos psicossociais } \\
\text { associados aos exames } \\
\text { de câncer de próstata em } \\
\text { idosos }\end{array}$ & Sousa MCP, et al. & $\begin{array}{l}\text { Investigar os aspectos psicossociais } \\
\text { associados aos exames de câncer de } \\
\text { próstata na visão do idoso. }\end{array}$ & $\begin{array}{l}\text { Exploratório } \\
\text { Descritivo }\end{array}$ & Qualitativa & 2014 \\
\hline 5 & $\begin{array}{l}\text { LILACS/ } \\
\text { SCIELO }\end{array}$ & $\begin{array}{l}\text { Revista Gaúcha } \\
\text { de Enferm. }\end{array}$ & $\begin{array}{c}\text { Um toque na } \\
\text { masculinidade: a } \\
\text { prevenção do câncer de } \\
\text { próstata em Gaúchos } \\
\text { tradicionalistas } \\
\end{array}$ & Souza LM, et al. & $\begin{array}{l}\text { Verificar a adesão desses gaúchos } \\
\text { tradicionalistas aos exames preventivos de } \\
\text { câncer de próstata, analisando, também, } \\
\text { quais variáveis influenciam na adesão ao } \\
\text { exame de toque retal. }\end{array}$ & Transversal & Quantitativo & 2011 \\
\hline
\end{tabular}

Fonte: Oliveira SL, et al., 2020. 
Os homens criam obstáculos relativos ao exame do toque retal, ocasionando um afastamento significativo deles nos serviços voltados para prevenção. Observa-se uma escassez destes nos locais de saúde, contribuindo para o aumento dos problemas encontrados relativos à saúde do homem. As medidas direcionadas pelos órgãos públicos existem para cuidar dos homens, com o intuito de diminuir ou eliminar os agravos de doenças que afetam o público masculino. Quando estes procuram as unidades de saúde, dificilmente os profissionais da área propiciam uma orientação para a realização do exame para detectar o câncer de próstata, dificultando o cuidado com o homem (SOUSA MCP, et al., 2014).

Vemos isso no estudo de Oliveira PSD, et al. (2015), onde estes são mais expostos às doenças crônicas, e vão à óbito com maior frequência em relação as mulheres, aumentando os riscos a sua saúde. Corroborando Ferreira DC, et al. (2015), focaliza que a prevenção é a alternativa eficaz para se obter um corpo saudável, com o objetivo de alcançar o bem-estar físico destes indivíduos.

De acordo com Freitas MEM, et al. (2015), o câncer de próstata é apontado como sendo a doença que mais afeta os homens depois dos 65 anos de idade, ficando apenas atrás do câncer de pele, e é a doença que mais leva os homens a morte, pois quando é diagnosticada, já está bastante avançada.

No texto de Sousa MCP, et al. (2014) é mencionado que é o tipo de câncer que mais atinge o homem, sendo considerado um problema de saúde pública. Com o aumento de novos casos, torna-se imprescindível um cuidado e uma atenção total para se alcançar a saúde plena do homem, envolvendo principalmente aqueles com idade para investigação desta doença.

Desse modo, Souza LM, et al. (2011) afirma que apesar deste problema, é preciso ações de prevenção, diagnóstico e tratamento para que a saúde do homem seja preservada. Essas ações mudam o estado de saúde dos participantes que buscam se cuidar.

Ferreira DC, et al. (2015) acrescenta que pode haver uma estimativa boa, se detectado no início da doença, o que também depende do estágio em que se encontra este tipo de câncer. No começo desta doença é possível viabilizar as medidas de controle, para que esta não se manifeste tão ameaçador à vida dos pacientes.

Nesse mesmo sentido, Oliveira PSD, et al. (2015), no seu estudo acredita que se identificados no início da doença, o câncer de próstata pode ter cura, obtendo uma expectativa positiva para que o homem viva com saúde e acredite que a recuperação é viável, e que vai melhorar seu estado de saúde.

O toque retal é um exame que consegue detectar a real situação e dimensão, da forma e do aspecto em que se encontra a próstata. Tem um custo baixo, todavia tem limitações para descobrir alguma alteração na próstata por completo (SOUZA LM, et al., 2011). Este exame é uma medida preventiva, e é um importante exame físico para rastrear o câncer de próstata (OLIVEIRA PSD, et al., 2015).

No estudo de Ferreira DC, et al. (2015), a partir dos 45 anos de idade, é priorizado que o homem que não apresentar sintomas precisa realizar o toque digital e o PSA anualmente, sendo que o toque é fundamental e eficaz, visto que avalia nódulos e verifica o comprometimento do local.

No texto de Sousa MCP, et al. (2014), é enfatizado que este exame por ser limitado, não sendo suficiente para identificar cerca de $40 \%$ a $50 \%$ dos nódulos. Apesar desse limite, observa-se que possui especificidade que garante a qualidade deste exame. Para Freitas MEM, et al. (2015), este exame mexe com a imaginário do homem, causando o distanciamento de realizá-lo, e colocando em risco seu bem-estar. A prevenção é uma possibilidade que esses homens têm de se cuidarem. Os fatores de recusa dos homens estão relacionados a aspectos culturais e emocionais nos quais estão atrelados à falta de informações, que compromete a realização do toque (OLIVEIRA PSD, et al. (2015).

$\mathrm{Na}$ análise de Freitas MEM, et al., (2015) a falta de informação, ou quando esta se apresenta de maneira errônea, criam "verdades" que são representadas por falsas crenças, e acabam atrapalhando o consentimento para se realizar os exames preventivos. O estudo de Ferreira DC, et al. (2015), salienta que acima dos 65 anos os homens estão propensos a desenvolverem o câncer de próstata. Boa parte dessa amostragem é analfabeta, ocasionando a desinformação da doença e dos exames de prevenção. A 
informação através de campanhas e de uma educação sistemática se faz necessária para esclarecer as dúvidas e motivar os homens ao exame.

Sousa MCP, et al. (2014), expõe que a falta de explicação antes do exame, e a falta de privacidade deste na hora de realizá-lo pode intervir no retorno ao especialista; pois em muitas situações os pacientes tem que fazer o exame em um ambiente com muitas pessoas e por profissionais do sexo oposto.

No estudo de Souza LM, et al. (2011) é destacado que o homem com escolaridade e renda elevada tem um cuidado maior com sua saúde, onde procura os serviços particulares e tem acesso a uma saúde diferenciada.

Quanto a questão do medo, Sousa MCP, et al. (2014) ressalta que entre as causas que interferem na busca da prevenção, está o receio de ser mexido em suas partes íntimas e, de sentir dor física ou simbólica, ou até mesmo o medo de identificar alguma doença séria em seu corpo. O desconhecimento sobre a existência de alguma doença gera uma falsa segurança, pois preferem não ter conhecimento de estarem doentes do que fazerem o exame e constatarem que possuem uma enfermidade. Infelizmente, esta é uma premissa que permeia o "imaginário" dos homens e que dita o seu comportamento.

Ferreira DC, et al. (2015), na sua perspectiva, o medo ou o preconceito estão ligados às emoções, mostrando assim porque a maioria dos homens demoram ou evitam a realização dos exames que rastreiam o câncer de próstata. Provavelmente isto acontece por falta de informação que deveria ser colocada em prática para buscarem o exame no momento adequado para cada homem. Outro item observado é o medo gerado de que a descoberta de um problema na próstata está ligada a possibilidade de impotência sexual.

Para Oliveira PSD, et al. (2015), a propagação do medo em relação a este exame acontece entre a própria população masculina, o que cria esses sentimentos das situações relacionadas a cultura, onde é desvalorizado o cuidado com a saúde.

Em relação ao preconceito, Freitas MEM, et al. (2015) reflete no seu estudo, que a homossexualidade está nos pensamentos dos homens como fator que impede o toque retal; apresentando a questão de que o homem que é homem não pode ser penetrado. Quando na verdade, o exame não muda seu comportamento como homem, mas passa a protegê-lo de uma doença que pode se agravar e atingir outros órgãos, impossibilitando que ele tenha uma vida longa e plena. Sob esse olhar, Souza LM, et al. (2011) descreve como a masculinidade interfere na realização do exame retal, principalmente nas zonas rurais, devido ao fato de lá os homens terem pensamentos antiquados, em que a masculinidade prevalece como barreira cultural. Além disso, os homens destes lugares, muitas vezes, estão sem serviços de saúde para fazer este exame. Este quadro é diferente dos homens que moram na zona urbana, os quais tem mais facilidade de buscar este exame, sendo que uma parcela significativa destes tem acesso nas instituições particulares.

Na compreensão de Freitas MEM, et al. (2015), o exame do toque retal abala a masculinidade, pois este requer um procedimento que vem de encontro a certas verdades já instaladas na mente dos homens, e exigirá que eles transponham a vergonha, o medo e outras situações que os deixam apavorados e inquietos, fazendo com que se sintam constrangidos e se recusem em realizar este exame. Fortalecendo o mesmo pensamento, Oliveira PSD, et al. (2015) divulga que o toque retal interfere no comprometimento da masculinidade, a partir do que representa na imaginação deste homem, mesmo que fique salientado a eficácia do exame pelos especialistas; assim, essa maneira de pensar impede na sua decisão de fazer.

No estudo de Freitas MEM, et al. (2015) evidencia-se que entre outros fatores da recusa, está que o médico não solicita o exame de toque retal durante as consultas. Este exame é importante, mas é insuficiente para detectar o câncer da próstata; sendo necessário esclarecerimentos para os homens. Outro fator colocado é a ausência de sintomas, em seu estágio inicial, em relação a algum possível problema da próstata; fazendo com que muitos achem que não há necessidades de realizar o exame, pois não sentem nenhum distúrbio. Contudo, há uma variedade de indicativos relevantes, mas que são fáceis de serem identificados, e que permitirão que o homem conheça a sua verdadeira situação, possibilitando que se tratem. 


\section{CONSIDERAÇÕES FINAIS}

Pela observação dos aspectos analisados, verificamos que os fatores da recusa são influenciados por motivos que se encontram no "imaginário" de muito dos homens. No que se refere à busca pelo exame do toque retal este homem não adere às possibilidades plausíveis deste cuidado. Percebeu-se nos estudos, que a falta de informação referente às vantagens de fazer o exame do toque retal, está ligada também a outros fatores levantados e averiguados na pesquisa, como o medo, o preconceito e a perda da masculinidade, que são consequências do desconhecimento do exame. Com base na leitura dos artigos selecionados, também constatamos que o enfermeiro tem importância em relação ao exame retal digital, pois este é pautado no embasamento científico, o que permite com que ele compreenda esses aspectos da recusa dentro da saúde pública; e também poderá agir de forma mais eficiente como agente de transformação desta realidade, pois além de estar mais perto da população, tem um papel primordial e profícuo no cenário da educação, que será a ação fundamental na prevenção do câncer de próstata.

\section{REFERÊNCIAS}

1. BARROS AL. Anamnese e Exame físico; avaliação diagnóstica de enfermagem no adulto. - 3. ed. - Porto Alegre: Artmed; 2016; 472p.

2. BRASIL, 2009. Ministério da Saúde. Secretaria de Atenção à Saúde. Departamento de Ações Programáticas e Estratégicas. Política Nacional de Atenção Integral à Saúde do Homem: princípios e diretrizes / Ministério da Saúde, Secretaria de Atenção à Saúde, Departamento de Ações Programáticas e Estratégicas - Brasília: Ministério da Saúde. Disponível em: http: //www.saude.gov.br/bvs. Acesso: 29 Mar de 2018.

3. CÂMARA RH, et al. Análise de conteúdo: da teoria à prática em pesquisas sociais aplicadas às organizações. Gerais: Revista Interinstitucional de Psicologia, 2013; 6, (2) 183.

4. FERREIRA DC, et al. Toque digital prostático: conhecimento e resistência masculina na terceira idade. Rev. Ciênc. Saúde Nova Esperança, 2015; 13 (2) 11-23.

5. FREITAS MEM, et al. Exame de toque retal: a percepção de homens quanto à sua realização. Rev. enferm. UFPI, 2015; 4 (4) 8-13.

6. INCA. 2018. Instituto Nacional do Câncer. Disponível em: http://www2.inca.gov.br. Acesso: 30 de Mar de 2018.

7. OLIVEIRA PSD, et al. Percepção dos homens sobre o exame toque retal. Revista de enfermagem UFPE on lineISSN: 1981-8963, 2015; 9, (5) 7760-7765.

8. SMELTZER SC, BARE BG. Brunner \& Suddarth. Tratado de Enfermagem Médico Cirúrgica. Rio de Janeiro: Guanabara Koogan; 2012; 1152p.

9. SOUZA LM, et al. Um toque na masculinidade: a prevenção do câncer de próstata em gaúchos tradicionalistas. Revista Gaúcha de Enfermagem, 2011; 32 (1) 151.

10. SOUSA MCP, et al. Aspectos psicossociais associados aos exames de câncer de próstata em idosos. Revista Interdisciplinar, 2014; 7 (3) 1-8.

11. SOUZA MT, et al. Revisão integrativa: o que é e como fazer. Einstein, 2010; 8 (1) 102-6. 